3. Chakravorthy S. COVID-19 in patients with sickle cell disease - a case series from a UK tertiary hospital. Haematologica. 2020;105(11): 2691-2693.

4. Frater JL, Zini G, d'Onofrio G, Rogers HJ. COVID-19 and the clinical hematology laboratory. Int J Lab Hematol. 2020;42(1):11-18.

5. Telfer P. Real-time national survey of COVID-19 in hemoglobinopathy and rare inherited anemia patients. Haematologica. 2020;105(11):2651-2654.

6. Hussain FA, Njoku FU, Saraf SL, Molokie RE, Gordeuk VR, Han J. COVID-19 infection in patients with sickle cell disease. $\mathrm{Br} J$ Haematol. 2020;189(5):851-852.

7. Beerkens F, John M, Puliafito B, Corbett V, Edwards C, Tremblay D. COVID-19 pneumonia as a cause of acute chest syndrome in an adult sickle cell patient. Am J Hematol. 2020;95(7):E154-E156.

8. Nur E, Gaartman AE, van Tuijn CFJ, Tang MW, Biemond BJ. Vasoocclusive crisis and acute chest syndrome in sickle cell disease due to 2019 novel coronavirus disease (COVID-19). Am J Hematol. 2020;95 (6):725-726.

9. Richardson S, Hirsch JS, Narasimhan M, et al. Presenting characteristics, comorbidities, and outcomes among 5700 patients hospitalized with COVID-19 in the New York city area. JAMA. 2020;323(20):2052-2059

10. Zhou F, Yu T, Du R, et al. Clinical course and risk factors for mortality of adult inpatients with COVID-19 in Wuhan, China: a retrospective cohort study. Lancet. 2020;395(10229):1054-1062

11. Wu C, Chen X, Cai Y, et al. Risk factors associated with acute respiratory distress syndrome and death in patients with Coronavirus disease 2019 pneumonia in Wuhan, China. JAMA Intern Med. 2020;180(7):934-943.

12. Shet AS, Thein SL. A growing population of older adults with sickle cell disease. Clin Geriatr Med. 2019;35(3):349-367.

13. Vichinsky EP, Neumayr LD, Earles AN, et al. Causes and outcomes of the acute chest syndrome in sickle cell disease. National Acute Chest Syndrome Study Group. N Engl J Med. 2000;342(25):1855-1865.

14. Connors JM, Levy JH. Thromboinflammation and the hypercoagula- bility of COVID-19. J Thromb Haemost. 2020;18(7):1559-1561.

15. (MMWR). CMaMWR. Hospitalization rates and characteristics of patients hospitalized with laboratory-confirmed Coronavirus disease 2019 - COVID-NET, 14 States, March 1-30, 2020. 2020 [cited; Available from: https://www.cdc.gov/mmwr].

16. Mehra MR, Desai SS, Ruschitzka F, Patel AN. Hydroxychloroquine or chloroquine with or without a macrolide for treatment of COVID-19: a multinational registry analysis. Lancet. 2020;S01406736(20):31180-31186.

17. Yazdany J, Kim AHJ. Use of Hydroxychloroquine and chloroquine during the COVID-19 pandemic: what every clinician should know. Ann Intern Med. 2020;172(11):754-755.

18. Naymagon L, Berwick S, Kessler A, Lancman G, Gidwani U, Troy K The emergence of methemoglobinemia amidst the COVID-19 pandemic. Am J Hematol. 2020;95(8):E196-E197.

19. Ware RE, Davis BR, Schultz WH, et al. Hydroxycarbamide versus chronic transfusion for maintenance of transcranial doppler flow velocities in children with sickle cell anaemia-TCD With Transfusions Changing to Hydroxyurea (TWiTCH): a multicentre, open-label, phase 3, non-inferiority trial. Lancet. 2016;387 (10019):661-670.

20. America SCDAo. Sickle cell disease and COVID-19: provider advisory. 2020 [cited; Available from: https://www.sicklecelldisease.org /files/sites/181/2020/03/4.10.20-MARAC-SCDAA-PROVIDERADVISORY-4-10-2020].

21. DeBaun MR. Initiating adjunct low dose-hydroxyurea therapy for stroke prevention in children with SCA during the COVID-19 pandemic. Blood. 2020;135(22):1997-1999.

22. Nickel RS, Margulies S, Frazer B, Luban NLC, Webb J. Combination dose-escalated hydroxyurea and transfusion: an approach to conserve blood during the COVID-19 pandemic. Blood. 2020;135(25): 2320-2322.

23. Chou ST, Alsawas M, Fasano RM, et al. American Society of Hematology 2020 guidelines for sickle cell disease: transfusion support. Blood Adv. 2020;4(2):327-355.

\title{
A CD205-directed antibody drug conjugate - lymphoma precision oncology or sophisticated chemotherapy?
}

\section{Damian T. Rieke ${ }^{1,2}$ and Ulrich Keller ${ }^{1,3}$}

${ }^{1}$ Department of Hematology, Oncology and Tumor Immunology, Campus Benjamin Franklin, Charité - Universitätsmedizin Berlin, 12203 Berlin; ${ }^{2}$ Berlin Institute of Health (BIH), 10178 Berlin and ${ }^{3}$ Max-Delbrück-Center for Molecular Medicine, 13092 Berlin, Germany

E-mail: ULRICH KELLER - ulrich.keller@charite.de

doi:10.3324/haematol.2020.261073

$\mathrm{P}$ recision oncology is expected to improve outcome of patients with malignant diseases by taking into account individual variability. ${ }^{1}$ This approach is strictly linked to the availability of a targeted treatment the efficacy of which depends on the presence of a molecular alteration, i.e., a predictive biomarker. This concept has been shown to be highly successful in well-defined subgroups of patients and has led to the histology-agnostic approval of drugs in solid tumors. ${ }^{2}$ Biomarker-stratified treatment has become first-line treatment in several solid tumors, such as non-small cell lung cancer. In many hematopoietic malignancies, including B-cell lymphomas, comparably higher cure rates and more treatment options have led to a more prognosis-oriented stratification of treatment. Here, prognostic biomarkers help to adjust treatment intensity to a cohort risk assessment. ${ }^{3}$ Together with improved prognostication of patients, a more refined diagnosis also helps with better treatment allocation.
Therefore, diagnostic biomarkers will help with the identification of defined disease subgroups. ${ }^{4}$ This might also correspond to differential outcome and/or response to treatment, and can therefore overlap with predictive and/or prognostic markers.

However, despite numerous advances in the understanding of cancer heterogeneity, not all diagnostic or prognostic stratifications will ultimately impact treatment and a number of patients will eventually have disease recurrence or progression. Therefore, the identification of novel treatment strategies is urgently required. The development of additional predictive biomarkers and corresponding drugs promises to improve outcome and limit toxicity. This advancement of precision oncology can be achieved in at least two ways: (i) the identification of the right treatment for given patients (as often tried in umbrella or unstratified precision oncology trials); ${ }^{5}$ or (ii) the identification of the right patient for a given treatment (as 
A

Antibody-drug conjugate

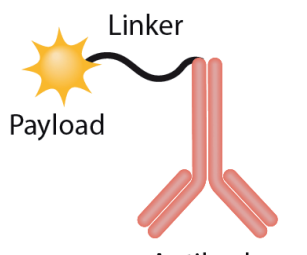

Antibody
B

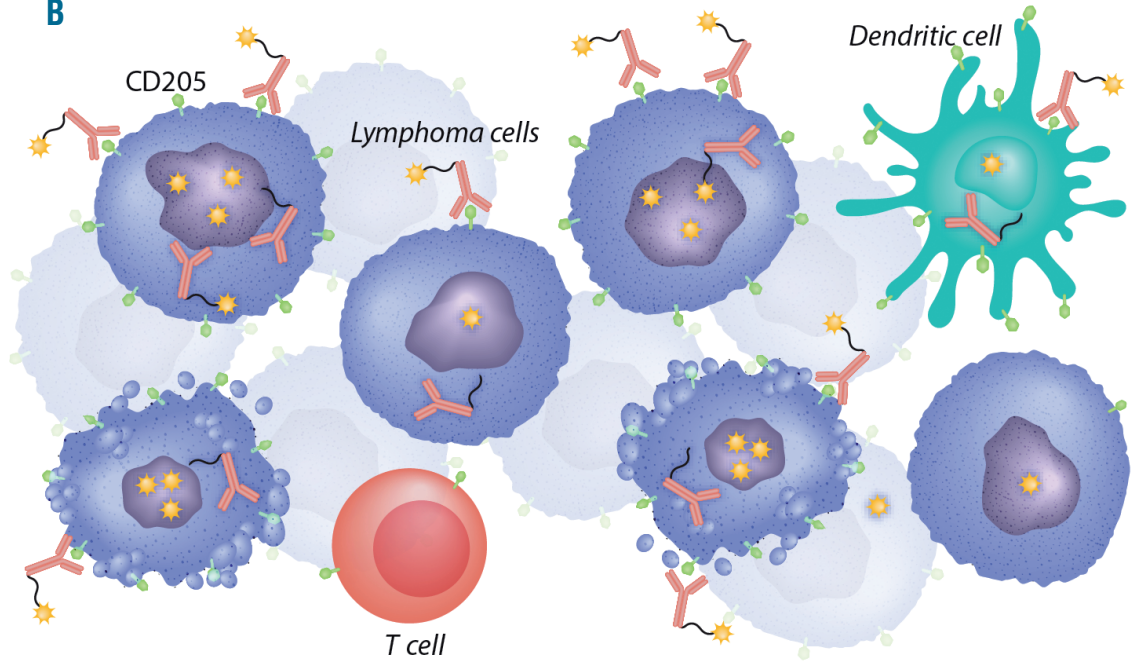

Figure 1. On- and off-target toxicities from antibody-drug conjugates (ADC) within the lymphoma microenvironment. (A) Schematic ADC, consisting of an antibody, a linker and the cytotoxic payload. Endocytosis of the ADC and linker cleavage will release the payload, resulting in cytotoxic effects. (B) On- and off-target cytotoxic effects from payload release with or without ADC internalization in a tumor microenvironment with abundant but heterogeneous expression of the target antigen. Payloads might be cleaved without endocytosis and/or permeate cell membranes to be taken up by bystander cells, thus facilitating drug efficacy even in tumor cells without adequate antigen expression. Toxic effects from microtubule-targeted compounds like maytansine on non-proliferating cells are typically reduced as compared to highly proliferating malignant cells (blue) with activated cell cycle checkpoints.

usually tried in basket trials). ${ }^{2}$

In this issue of Haematologica, Gaudio et al. show the efficacy of an antibody drug conjugate (ADC) targeting CD205 in lymphoma models. ${ }^{6}$ Importantly, the efficacy of this new drug, MEN1309/OBT076, was significantly associated with cell surface expression of the target CD205 in B-cell lymphoma cell lines. Furthermore, cytotoxicity of MEN1309/OBT076 was reduced with the introduction of a competitive CD205 antibody. These findings underline the dependence of drug efficacy on target availability, suggesting the potential of CD205 expression as a predictive biomarker. In an in vitro screen, MEN1309/OBT076 efficacy did not depend on B-cell lymphoma subtype. Together with previous preclinical results in CD205-positive triplenegative breast, pancreatic and bladder cancer cell lines and xenografts, ${ }^{7}$ this creates a virtual preclinical basket trial which now awaits clinical validation.

However, several questions remain to be answered for the clinical development of MEN1309/OBT076 in lymphoma. In addition to its predictive significance in preclinical models (that, as we have said, still needs to be validated in clinical trials), the biological function of CD205 is surprisingly unclear. Previous reports show that CD205 is expressed on leukocytes, mainly dendritic cells and monocytes $^{8,9}$ with a role in endocytosis and the recognition of apoptotic and necrotic cells. . $^{11}$ The role in lymphomagenesis remains even less understood. A fusion protein involving CD205 was identified in Hodgkin lymphoma ${ }^{12}$ but also in normal dendritic cell maturation. ${ }^{10}$ However, improved characterization of the biological role of this protein remains vital to understanding its implications in the clinic (as a prognostic biomarker in the identification of adequate clinical settings for the introduction of a novel drug) as well as a diagnostic biomarker. If CD205 helps in defining a biologically distinct subgroup within lymphomas, this would be particularly relevant for the identi- fication of rational combination partners, of which two, venetoclax and rituximab, showed promising signals in the study by Gaudio et al. ${ }^{6}$ However, the broad expression of the antigen with a moderate to intense CD205 expression in $20-50 \%$ of tested lymphoma samples, and an overall expression of the antigen in more than $70 \%$ of samples, makes it unlikely that CD205 adequately reflects lymphoma heterogeneity, and stability of CD205 protein expression needs to be validated. Stable expression of the antigen is probably linked to continued efficacy of the drug, following the successful examples of other ADC such as brentuximab vedotin (targeting CD30), ${ }^{13}$ polatuzumab vedotin (targeting CD79b), ${ }^{14}$ or trastuzumab emtansine (targeting HER2). ${ }^{15}$ Efficacy of trastuzumab emtansine could still be demonstrated despite previous HER2-directed therapies and retreatment with brentuximab vedotin showed responses in the majority of patients that had relapsed after initial response to the same drug. ${ }^{16}$ In the work by Gaudio et al. ${ }^{6}$ a rechallenge with MEN1309/OBT076 was also sufficient to induce remission in the only xenograft model with tumor regrowth after a first dose of the ADC. These and other data support the further development of $A D C$ as powerful tools for the targeted delivery of cytotoxic drugs. Interestingly, neither resistance to CD30-directed ADC or to HER2-directed ADC seems to be mediated by a loss of target antigen expression but rather by dysfunctional intracellular metabolism of the payload. ${ }^{17,18}$ Again, the continued expression of CD205 even under therapeutic pressure remains to be determined and is probably linked to its biological role. Expression of the antigen is also important to predict toxicities in human trials. Even though previous work has not identified relevant toxicities in cynomolgus monkeys, ${ }^{7}$ potential risks to humans will also depend on disease characteristics and are still to be determined in ongoing clinical trials. 
Since CD205 is broadly expressed in lymphoma cells and leukocytes, target antigen expression can be expected in most tumors and/or their microenvironment. Since MEN1309/OBT076 is designed with a cleavable linker, payload release does not necessarily depend on ADC endocytosis, thus facilitating bystander killing and off-target toxicity. ${ }^{19}$ In the case of a broadly CD205 expressing tumor microenvironment, an adequate on-tumor efficacy could therefore be expected even without adequate ontarget effects and ubiquitous antigen expression on lymphoma cells (Figure 1). In this case, the novel ADC could rather act as a more sophisticated chemotherapy-delivery system and CD205 expression will not allow adequate patient selection. Even in this case, the drug might still prove useful in lymphoma therapy alone or in combination. However, the integration of this novel agent into current treatment schedules might become more difficult.

In conclusion, the work by Gaudio et al. ${ }^{6}$ shows the activity of the anti-CD205 ADC MEN1309/OBT076 in preclinical CD205-positive lymphoma models that warrants further clinical investigation. The development of a biomarker-drug combination allows for a targeted application of this drug in clinical trials. However, additional preclinical and translational work is required to shed light on the role of CD205 in lymphomagenesis. This is important for the rational development of this treatment as monotherapy, but also, and in particular, as a part of combination therapy. ADC continue to be important components of tumor therapy that could sometimes find a place between precision oncology and refined chemotherapy.

\section{Funding}

UK is supported by Deutsche Forschungsgemeinschaft, Deutsche Krebshilfe, Stiftung Charité, Wilhelm Sander-Stiftung and the Berlin Institute of Health.

\section{Acknowledgments}

DTR is a participant in the Berlin Institute of Health - Charite Clinical Scientist Program funded by the Charite Universitätsmedizin Berlin and the Berlin Institute of Health.

\section{References}

1. Collins FS, Varmus H. A new initiative on precision medicine. N Engl J Med. 2015;372(9):793-795.

2. Drilon A, Laetsch TW, Kummar S, et al. Efficacy of larotrectinib in
TRK fusion-positive cancers in adults and children. N Engl J Med. 2018;378(8):731-739.

3. Poeschel V, Held G, Ziepert M, et al. Four versus six cycles of CHOP chemotherapy in combination with six applications of rituximab in patients with aggressive B-cell lymphoma with favourable prognosis (FLYER): a randomised, phase 3, non-inferiority trial. Lancet. 2020;394(10216):2271-2281

4. Sukswai N, Lyapichev K, Khoury JD, Medeiros LJ. Diffuse large Bcell lymphoma variants: an update. Pathology. 2020;52(1):53-67.

5. Lamping M, Benary M, Leyvraz S, et al. Support of a molecular tumour board by an evidence-based decision management system for precision oncology. Eur J Cancer. 2020;127:41-51.

6. Gaudio E, Tarantelli C, Spriano F, et al. Targeting CD205 with the antibody drug conjugate MEN1309/OBT076 is an active new therapeutic strategy in lymphoma models. Haematologica. 2020;105(11): 2584-2591.

7. Merlino G, Fiascarelli A, Bigioni M, et al. MEN1309/OBT076, a firstin-class antibody-drug conjugate targeting CD205 in solid tumors. Mol Cancer Ther. 2019;18(9):1533-1543

8. Kato M, McDonald KJ, Khan S, et al. Expression of human DEC-205 (CD205) multilectin receptor on leukocytes. Int Immunol. 2006;18(6):857-869.

9. Fukaya T, Takagi H, Uto T, Arimura K, Sato K. Analysis of DC functions using CD205-DTR knock-in mice. Methods Mol Biol. 2016;1423:291-308.

10. Butler M, Morel AS, Jordan WJ, et al. Altered expression and endocytic function of CD205 in human dendritic cells, and detection of a CD205-DCL-1 fusion protein upon dendritic cell maturation. Immunology. 2007;120(3):362-371.

11. Cao L, Shi X, Chang H, Zhang Q, He Y. pH-Dependent recognition of apoptotic and necrotic cells by the human dendritic cell receptor DEC205. Proc Natl Acad Sci U S A. 2015;112(23):7237-7242.

12. Kato M, Khan S, Gonzalez N, et al. Hodgkin's lymphoma cell lines express a fusion protein encoded by intergenically spliced mRNA for the multilectin receptor DEC-205 (CD205) and a novel C-type lectin receptor DCL-1. J Biol Chem. 2003;278(36):34035-34041.

13. Younes A, Bartlett NL, Leonard JP, et al. Brentuximab vedotin (SGN35) for relapsed CD30-positive lymphomas. N Engl J Med. 2010;363(19):1812-1821.

14. Sehn LH, Herrera AF, Flowers CR, et al. Polatuzumab vedotin in relapsed or refractory diffuse large B-cell lymphoma. J Clin Oncol. 2020;38(2):155-165.

15. Burris HA 3rd, Rugo HS, Vukelja SJ, et al. Phase II study of the antibody drug conjugate trastuzumab-DM1 for the treatment of human epidermal growth factor receptor 2 (HER2)-positive breast cancer after prior HER2-directed therapy. J Clin Oncol. 2011;29(4):398-405.

16. Bartlett NL, Chen R, Fanale MA, et al. Retreatment with brentuximab vedotin in patients with CD30-positive hematologic malignancies. J Hematol Oncol. 2014;7:24.

17. Hunter FW, Barker HR, Lipert B, et al. Mechanisms of resistance to trastuzumab emtansine (T-DM1) in HER2-positive breast cancer. Br J Cancer. 2020;122(5):603-612.

18. Nathwani N, Krishnan AY, Huang Q, et al. Persistence of CD30 expression in Hodgkin lymphoma following brentuximab vedotin (SGN-35) treatment failure. Leuk Lymphoma. 2012;53(10):2051-2053.

19. Hoffmann RM, Coumbe BGT, Josephs DH, et al. Antibody structure and engineering considerations for the design and function of antibody drug conjugates (ADCs). Oncoimmunology. 2018;7(3): e1395127. 